\title{
Emotion may predict susceptibility to fake news but emotion regulation does not seem to help
}

Bence Bago $^{1 *}$, Leah R. Rosenzweig ${ }^{2}$, Adam J. Berinsky ${ }^{3}$, David G. Rand ${ }^{4,5}$

${ }^{1}$ Institute for Advanced Study in Toulouse, University of Toulouse 1 - Capitole.

${ }^{2}$ Stanford University

${ }^{3}$ Mitsui Professor, Department of Political Science, Massachusetts Institute of Technology

${ }^{4}$ Sloan School, Massachusetts Institute of Technology

${ }^{5}$ Department of Brain and Cognitive Sciences, Massachusetts Institute of Technology

*Corresponding author: Bence Bago, Institute for Advanced Study in Toulouse, University of Toulouse 1 -

Capitole, 21 Allée de Brienne, 31015 Toulouse, France, e-mail: bencebagok@gmail.com 


\begin{abstract}
Misinformation is a serious concern for societies across the globe. To design effective interventions to combat the belief in and spread of misinformation, we must understand which psychological processes influence susceptibility to misinformation. This paper tests the widely assumed -- but largely untested -- claim that people are worse at identifying true versus false headlines when the headlines are emotionally provocative. Consistent with this proposal, we found correlational evidence that overall emotional response at the headline level is associated with diminished truth discernment, except for experienced anger which was associated with increased truth discernment. A second set of studies tested a popular emotion regulation intervention where people were asked to apply either emotional suppression or emotion reappraisal techniques when considering the veracity of several headlines. In contrast to the correlation results, we found no evidence that emotion regulation helped people distinguish false from true news headlines.
\end{abstract}




\section{Introduction}

Fake news - news that mimics true media content, but is completely invented - has become a major source of concern in society, and focus of academic research (Berinsky, 2017; Grinberg et al., 2019; Lazer et al., 2018; McLaughlin, 2018; Pennycook et al., 2021; Pennycook \& Rand, 2021; Vosoughi et al., 2018). Besides the practical importance of decreasing belief in misinformation, researching fake news can help us understand deeper theoretical questions regarding how reasoning goes awry and makes people believe in false statements and how they judge the veracity of information (Brashier \& Marsh, 2020).

This paper sheds light on this issue by investigating emotional processes behind belief in fake news. News - and fake news in particular - can be highly emotionally evocative, which may contribute to its spread online (Brady et al., 2017). It has been suggested in various popular press outlets that people fall for fake news precisely because of its emotionally provocative nature (Barr, 2019; Lynch, 2019). This idea also logically follows from previous theoretical work on misinformation and deliberation ${ }^{1}$ (Bago et al., 2020; Pennycook \& Rand, 2019). Taking a dual-process perspective on misinformation, Pennycook and Rand (2019) concluded that when people engage in deliberation, they are more likely to correctly distinguish fake content from real, compared to when they rely on their intuitions. Importantly, emotions have been widely identified as a potential cause for erroneous intuitive responses (and people's lack of deliberation) in many contexts ${ }^{2}$ (Holland et al., 2012; Lerner $\&$ Tiedens, 2006; Slovic et al., 2007).

Despite the intuitive appeal of this account, there is little direct supporting empirical evidence. There is some evidence that emotions make people believe in false, but not true, headlines (Martel et al., 2020); but this work only measured mood prior to exposure to the news, and manipulated participants' overall reliance on emotion, rather than examining the emotions induced by the information content itself. Although these findings suggest that emotionality of headlines might cause people to preferentially believe in false headlines, they do not provide direct evidence of this claim.

Here, we address this question. In a first study, we directly examine whether experienced emotions are predictive of susceptibility to misinformation, using correlational data. We found evidence that the majority of emotions are associated with diminished truth discernment; that is, people who feel an emotion -- any emotion other than anger -- after

\footnotetext{
${ }^{1}$ We refer to deliberation from the dual process perspective (Evans \& Stanovich, 2013); that is, a process that requires working memory and cognitive control to operate.

${ }^{2}$ When we refer to "emotions", we always refer to the 6 basic emotions (Ekman, 1992), as they are frequently associated with intuition, or identified as intuitive processes themselves (Evans, 2012), unlike more complex emotions such as guilt or pride.
} 
reading a headline are more likely to erroneously believe that false headlines are true, compared to those who felt no emotion.

Having established that experiencing emotion is related to belief in fake news, in three additional studies we tested an intervention to make people inhibit their emotional response, thus opening the door for deliberation. In the first of the three experiments, we experimentally induce subjects to control their emotions by inducing them to use two well-documented emotion regulation techniques: suppression and cognitive reappraisal. These emotion regulation techniques are quite popular in the literature and have been used to alter decision outcomes in many tasks in which responding is highly influenced by emotions (Feinberg et al., 2012; van't Wout et al., 2010). After finding some evidence for the effectiveness of emotion suppression against belief in fake news, we conducted 2 further studies to try to replicate the findings both with older (Study 3) and newer (Study 4) headlines. However, the replication studies were unsuccessful. Overall, we find little credible evidence that emotion regulation techniques help reduce belief in fake news.

\section{Study 1}

\section{Methods}

Participants. In total, 998 participants (405 females, 579 males and 7 others, Mean age $=$ 38.4 years, $\mathrm{SD}=10.9$ years) took part in this study, who were recruited through MTurk, and completed the experiment online. In total, 444 people were Liberals, and 307 were Conservatives, 221 were moderates, and 24 "haven't thought much about it."

\section{Materials \& Procedure}

News headlines. We used a pool of 24 items, taken from Pennycook and Rand (2018), half of which were real (true) and the other fake (false). Moreover, half of the items were Republican-consistent and the other half were Democrat-consistent items, based on a pretest. Participants were presented with 16 randomly selected headlines altogether; 4 from each category (i.e., Republican-consistent fake, Republican-consistent real, Democrat-consistent fake, Democrat-consistent real). Headlines were presented in a random order. For each headline, participants were asked the following question: "Do you think this headline accurately describes an event that actually happened?" with the response options "Yes/No". Afterwards, participants were also asked about their intention to read more about the story and share it on social media: "If it was possible, would you click on this headline to read the full story? (Yes/No)" and "If you were to see the above article online, would you share it? (Yes/No)". The headlines are available in the OSF page of the project. 
Emotion measures. We measured the 6 basic emotions (Ekman, 1992): fear, anger, disgust, happiness, sadness, surprise. To measure emotional responses to each headline, we asked participants to select the icon(s) that best represented their emotional state when they read the headline, and then we presented them with 6 icons representing each distinctive emotion and a "None/Other" option. To rule out potential biases arising from verbal reports of emotional reactions, some researchers have used a set of facial expressions representing each distinctive emotion (see, for example: Gutierrez \& Giner-Sorolla, 2007). However, the recognition of these facial expressions comes with its own set of potential biases, for example, expressions are more likely to be misjudged if the ethnicity of the participant and the person in the picture are different (Elfenbein \& Ambady, 2002), while gaze patterns and subjective attractiveness could also moderate response patterns (Langner et al., 2010). Hence, instead of facial expressions, we presented emoticons - widely used in online communications - to avoid these potential biases, and to generate an approach which could be used subsequently for valid cross-cultural comparisons. Figure 1 presents our selection of emoticons. To make sure that participants indeed correctly recognize these emoticons, we conducted a pre-test with 100 participants. In this pre-test, participants were asked to indicate which emotion is represented by a given emoticon. They selected their response from a list: "anger", "fear", "disgust", "surprise", "happy", "sad", "other, please specify:" (for the validity of this methodology, see: Frank \& Stennett, 2001). Our results showed that the recognition rate for each one of our emotions were above $80 \%$ (anger: $97 \%$, fear: $92 \%$, disgust: $81 \%$, surprise: $94 \%$, happiness: $100 \%$, sadness: $97 \%$ ), indicating that our scale is a valid measure of emotions (e.g., accuracy rates are comparable - and sometimes even better than on the widely used Radboud Faces Dataset; Langner et al., 2010).
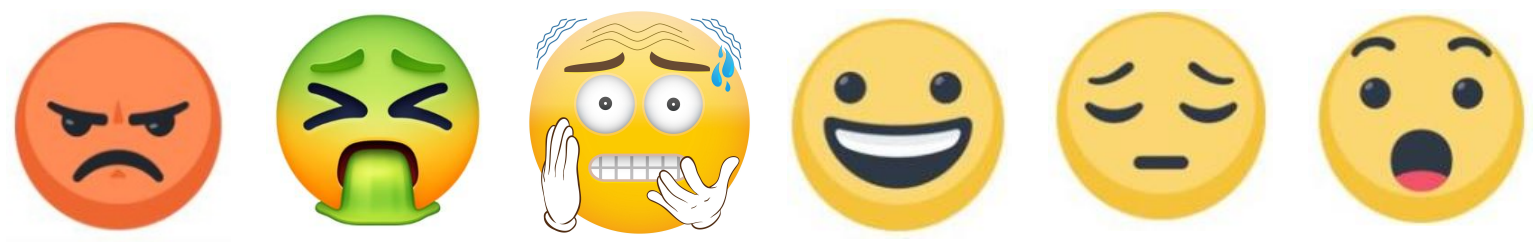

Figure 1. The emoticons participants were presented with, each represents one particular emotion. From left to right: anger, disgust, fear, happiness, sadness, surprise. Participants were also presented with a "Neutral/None of these" option. These emojis were taken from Facebook, with the exception of fear; this was additionally designed by a graphics designer.

Procedure. In the experiment, we randomized the order in which emoticons were presented for each participant, but kept the same order for a given participant throughout the experiment. After a participant selected the emotions she experienced, she was presented with the emoticons she selected and was asked to rate how much she felt the selected emotions on a scale from 1 (not very strongly) to 7 (very strongly). 
Half of the participants received the emotion question first ${ }^{3}$, before the accuracy question; the other half received these two questions in the reverse order. At the end of each trial, subjects received the question regarding clicking and sharing (in this order).

After the instructions, participants received one practice problem to make sure they understood the procedure. At the very beginning of the practice problem, participants were also told: "In the following question, you will be first asked about what emotions you experienced when you first read the headline. Each of the six emojis you will see represents one distinctive emotional expression. Select as many as you want." After the experiment participants were presented with the "emotion recognition test" (for description see: emotion recognition pre-test), and they filled out 7 items of the Cognitive Reflection Test (Frederick, 2005; Thomson \& Oppenheimer, 2016), and the Emotion Regulation Questionnaire (Gross \& John, 2003). At the end of the questionnaire, we asked various questions regarding demographics and political orientation.

We classified participants into "Liberal" or "Conservative" based on the question: "Generally speaking, do you usually think of yourself as a liberal, a conservative, a moderate, or haven't you thought much about this? (Liberal/Conservative/Moderate/Haven't thought much about it) ". If they selected "Conservative" we considered Republican-consistent headlines "politically concordant" and Democrat-consistent headlines as "politically discordant"; if they selected "Liberal", we considered Democrat-consistent headlines "politically concordant" and Republican-consistent headlines as "politically discordant". For participants who indicated "Moderate" or "Haven't thought much about it", all headlines were coded as politically neutral. We use subject ideology rather than partisanship to classify concordance because subject partisanship was not collected due to a programming error.

Statistical models. We used mixed effect logistic regression models with items and subjects as random intercepts, and presence of emotion $(0=$ no, $1=$ yes $)$, veracity of headlines $(-0.5=$ fake, $0.5=$ real $)$, political concordance $(-0.5=$ discordant, $0=$ neutral, $0.5=$ concordant $)$ as fixed effects. We always tried to fit the maximal model (using all possible random slopes) and if that didn't converge or caused singularity issues, we decreased model complexity. If there were more than one model that converged without a problem with the same level of random structure complexity, we used AIC to decide which model has the better fit and reported the results of that model.

All data are available at the OSF page of the project: osf.io/pn8ja

\footnotetext{
${ }^{3}$ We added presentation order to the most critical model, in which we analyze the effect of emotion, headline veracity and concordance on perceived accuracies, and found no significant main or interacting effect of order $(p>0.07)$. Presentation order however did have a main effect on how much emotion was experienced, $b=0.6, p=0.0002$; people who had to report emotions first, before accuracy, reported more emotions in general $(73 \%)$, than people who received the accuracy question first $(65.6 \%)$. This suggests that emotions fade; participants are more likely to be aware of them and report them right when they experience them.
} 


\section{Results}

In $69.3 \%$ of trials, people reported experiencing at least one of the six basic emotions (See Figure 2 for a summary). This result suggests that people tended to experience emotions in a majority of headlines. We found no evidence that headline veracity is associated with the presence of emotions in a given headline, $b=-0.14, p=0.47,(68.7 \%$ emotional on real, $69.9 \%$ emotional on fake), and we also found no relationship with the headline's political concordance, $\mathrm{b}=0.23, \mathrm{p}=0.15) 72.9 \$,$% emotional on concordant, 69.9 \%$ emotional on discordant). The interaction of veracity and concordance was not significant, $b=0.12, p=$ 0.71. That is, the concordance relationship with experiencing emotion is independent from the headline's actual veracity. Second, we tested whether their average emotional response (i.e., how much they experienced the emotions they said they experienced) varied by the headline's veracity or concordance. We found no significant association with concordance, $b$ $=0.05, p=0.42$, veracity, $b=-1.03, p=0.7$, or their interaction, $b=0.04, p=0.72$. This suggests that the average strength of an emotion after seeing a particular headline does not depend on the headline's veracity or political concordance, but people are more likely to experience emotions after seeing concordant headlines.

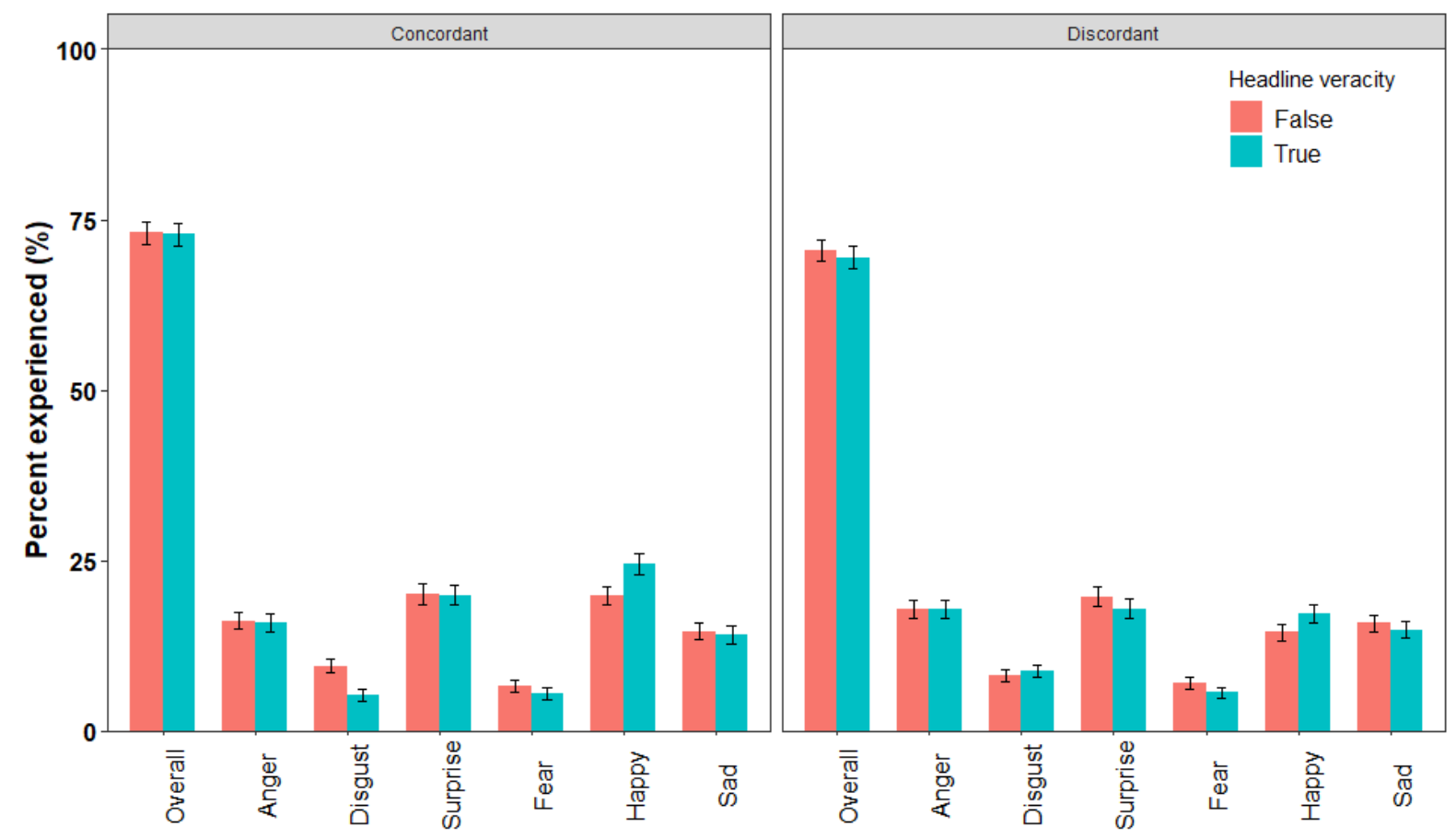

Figure 2. Figure shows percent of headlines in which a given emotion was experienced.

We then turned to our main question of interest - how experiencing emotion corresponds with accuracy perceptions (see Figure 3 for a summary). When we regressed perceived accuracy on veracity, concordance and emotion, the model allowed for the variation of emotion, concordance and veracity over subjects and emotion and concordance over items. We found a significant relationship with headline veracity; $b=2.3, p<0.0001$, the presence of any emotion, $b=1.3, p<0.0001$, (i.e., people are more to believe in 
headlines when experiencing emotions) and an effect of concordance, $b=0.45, p=0.003$. We also found a significant interaction between emotion and headline veracity, $b=-0.4, p=0.02$, such that truth discernment between true and false was worse for headlines where participants experienced emotion than those where participants did not experience emotion. In order to get a better understanding of the two way interaction between veracity and emotion, we analysed true and false headlines separately from each other; there is a positive association with emotion for true headlines (model allowed for the variation of emotions over subjects and items), $\mathrm{b}=1.18, \mathrm{p}<0.0001$, and there is also a positive association with emotion for fake headlines (model allowed for the variation of emotions over subjects), with a larger effect size than for true headlines, $\mathrm{b}=1.44, \mathrm{p}<0.0001$. No other interactions were significant in this model, $\mathrm{p}>0.1$.

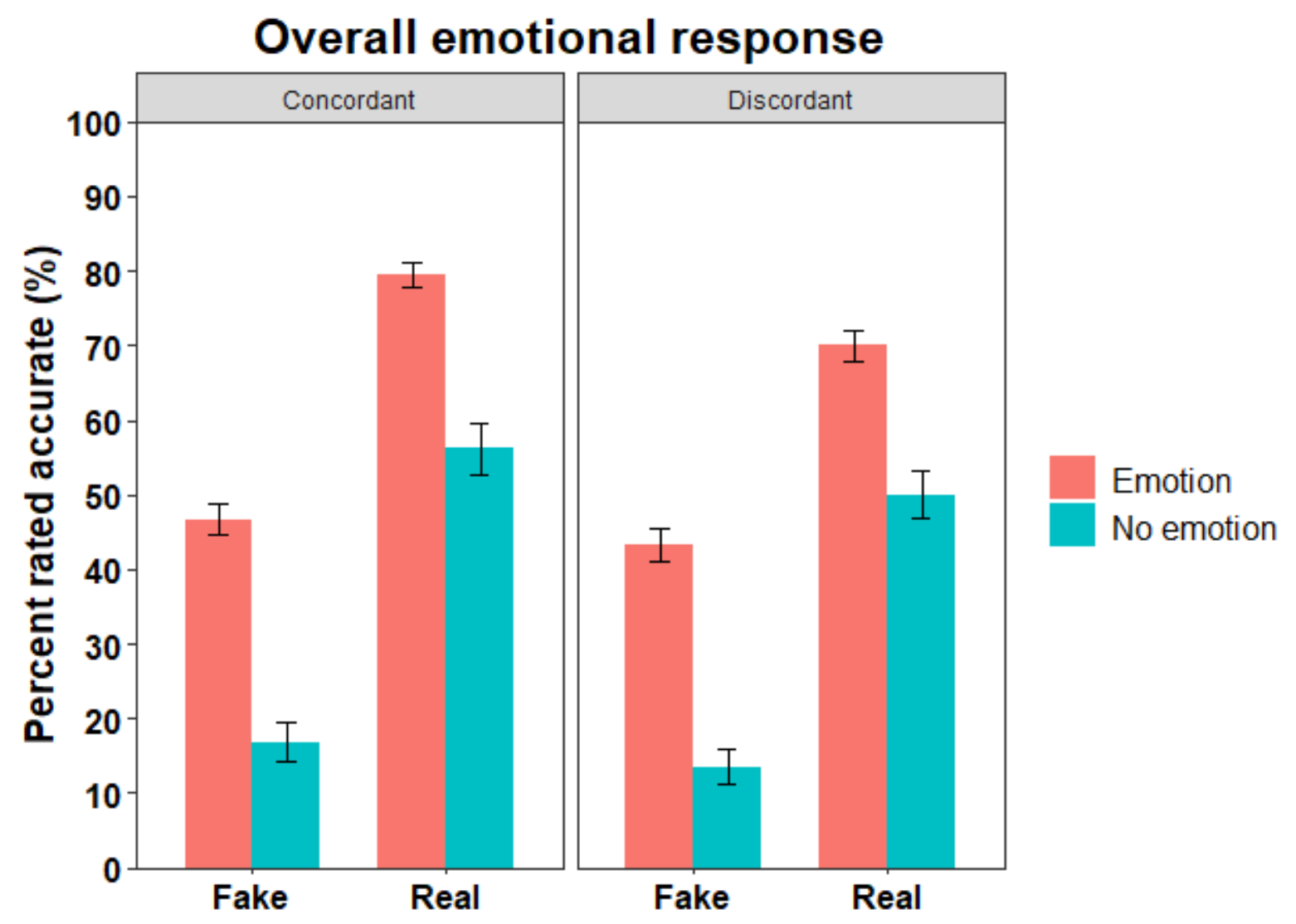

Figure 3. Figure shows perceived accuracy averages as a function of headline veracity and political concordance, when participants experienced any of the 6 basic emotions in a given headline, vs when they did not. Error bars are 95\% confidence intervals.

An individual analysis of each separate emotion can be found in the Supplementary Materials. To summarize, happiness (on discordant headlines) and disgust were associated with significantly decreased truth discernment, and sadness, surprise and fear had a non-significant (but negative in sign) association with discernment. Anger, conversely, was 
associated with significantly increased discernment but only on discordant headlines (SI for further details).

Sharing and clicking intentions. Exploratively, we also conducted an analysis using sharing and clicking intentions as dependent variables. Figure 4 summarizes results. This model allowed for the variation of emotion, concordance and veracity over subjects and emotion over items. We found that emotions had a main effect on sharing intentions, $b=$ $5.26, \mathrm{p}<0.0001$, (people are more likely to share emotion-invoking content), as well as concordance, $\mathrm{b}=1.39, \mathrm{p}<0.0001$, (people are more likely to share concordant information) and veracity, $\mathrm{b}=1.39, \mathrm{p}<0.0001$ (people are more likely to share true headlines). The three-way interaction was also significant, $b=1.35, \mathrm{p}=0.02$. To understand this three way interaction, we analysed concordant and discordant headlines separately. We find no significant veracity and emotion interaction in either case, however, the direction of the coefficients suggests that emotion worsens sharing discernment in discordant headlines (this model allowed for the variation of emotion over subjects and items and the variation of veracity over subjects), $b=-0.99, p=0.08$, while it increases sharing discernment in concordant headlines (this model allowed for the variation of emotion over subjects and items), $b=0.5, p=0.35$, resulting in a significant three-way interaction.

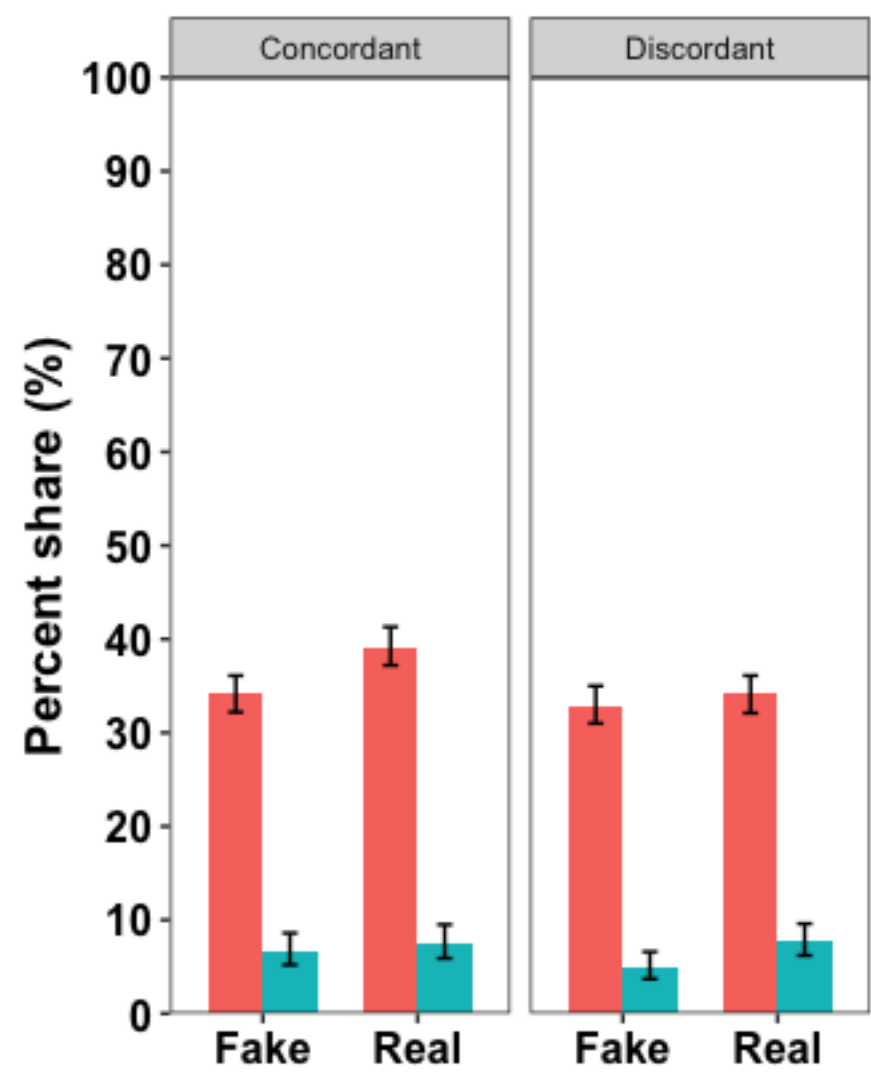

Any emotion No emotion 
Figure 4. Figure shows sharing intention averages as a function of headline veracity and political concordance, when participants experienced any of the 6 basic emotions in a given headline, vs when they did not. Error bars are $95 \%$ confidence intervals.

Figure 5 summarizes results for clicking intentions. This model allowed for the variation of veracity, concordance and emotions over subjects, and the variation of emotion over items. We found that emotion had a significant main effect, $b=2.04, p<0.0001$, (people are more likely to click on emotional content), as well as veracity, $b=1.22, p<0.0001$, (people are more likely to click on true headlines) and concordance, $b=0.56, p=0.0002$ (people are more likely to click on concordant rather than on discordant headlines). We also found a significant interaction between veracity and emotion, suggesting that experiencing emotion was negatively associated with clicking discernment, $\mathrm{b}=-0.46, \mathrm{p}=0.002$, and $\mathrm{a}$ three-way interaction among concordance, veracity and emotion, $b=0.61, p=0.04$, suggesting that emotion was associated with worse clicking discernment especially on discordant headlines.

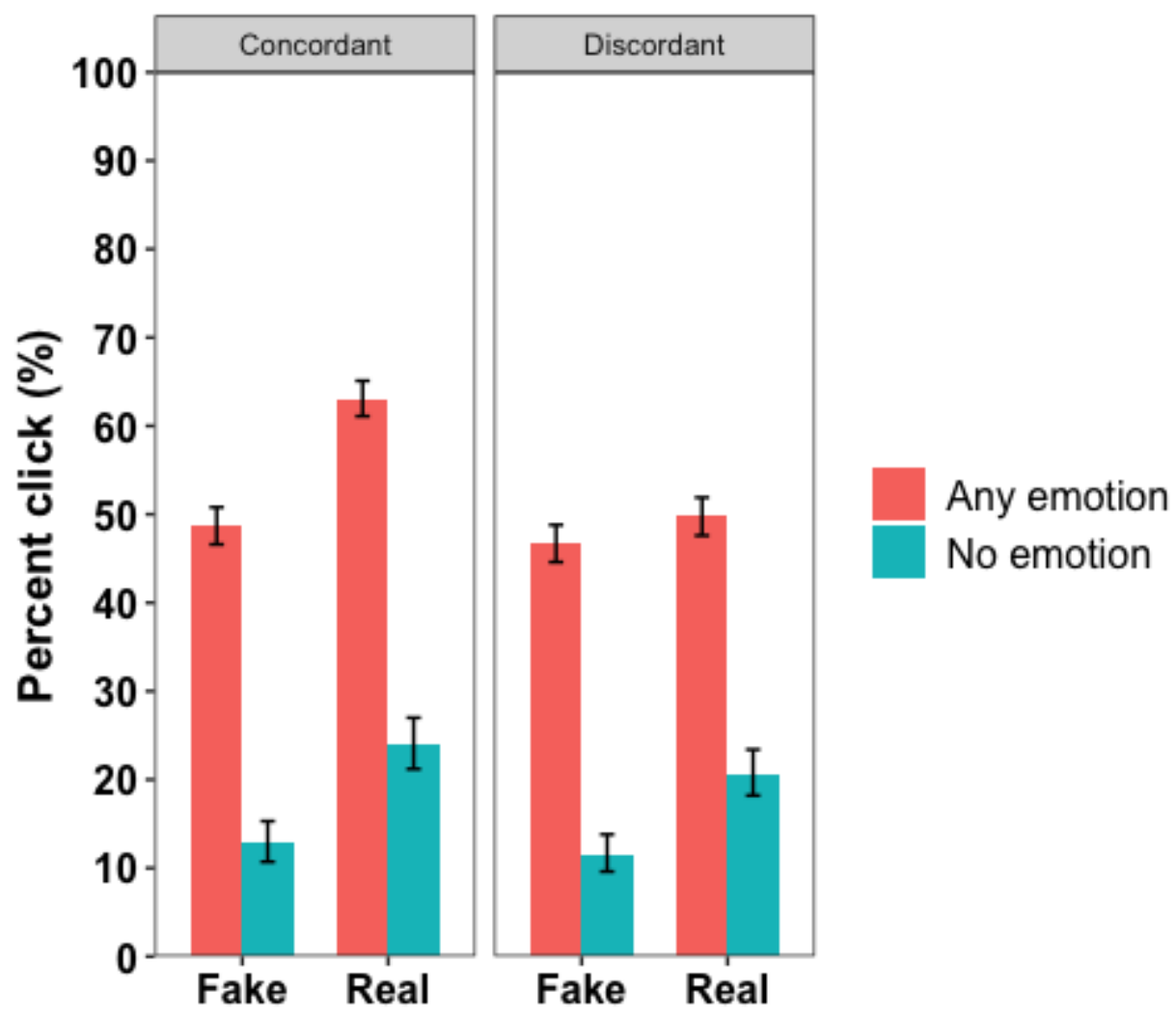

Figure 5. Figure shows clicking intention averages as a function of headline veracity and political concordance, when participants experienced any of the 6 basic emotions in a given headline, vs when they did not. Error bars are 95\% confidence intervals. 


\section{Discussion}

Our data suggest that having an emotional response to a given headline is typically associated with greater belief for both true and false headlines - and with diminished truth discernment(although anger was associated with increased discernment on discordant headlines). Note that it is possible that the association between truth discernment and emotions could have been caused by a ceiling effect. That is, emotions might have simply increased overall belief in headlines, but since belief in true headlines is already comparatively high, there was less room for belief in true headlines to increase. This (and the correlational nature of the study) prevents us from drawing any conclusions about causal effects of emotion on truth discernment.

In Study 2, we build on this by testing the effectiveness of interventions designed to reduce emotional responses in order to improve discernment. This should also inform us about the causal relationship between emotions and truth discernment.

Emotion regulation strategies are often employed to achieve a different, often less (negative) emotional state. The literature offers several emotion regulation strategies (for a review, see: Gross, 1998). We focus on two well-studied strategies: cognitive reappraisal and emotion suppression. Reappraisal is defined as: "interpreting potentially emotion-relevant stimuli in unemotional terms," while emotion suppression is "inhibiting emotion-expressive behavior while emotionally aroused" (Gross, 1998, p. 226). These strategies have different effects on our emotional, physiological and neural states (Gross, 2002). Most importantly, cognitive reappraisal has been shown to be effective at inducing deliberation, in the presence of emotional stimuli (Feinberg et al., 2012; van't Wout et al., 2010). So far, there has been no strategy identified that induces deliberation when people judge the truth/falsity of news headlines; all manipulations that have been applied to test the "lack of deliberation" hypothesis were negative in the sense that they induced intuition, and decreased deliberation (Bago et al., 2020, Martel et al., 2019; e.g., people were asked to rely on their emotions, or were forced to make an intuitive decision). Hence, if we find a manipulation that induces deliberation, we can increase peoples' discernment performance. Such manipulations could be directly applicable in practice to fight against fake news. ${ }^{4}$

In Study 1, we measured the habitual use of these two emotion regulation techniques. We separately added them to the regression models, together with concordance and headline veracity. We found that when cognitive reappraisal was included in the models, there was a main effect of headline veracity; $b=1.78, p<0.0001$, and reappraisal, $b=0.12, p=0.004$, but there interaction between them was non-significant (although positive in sign), $b=0.05, p$ $=0.1$. Thus we do not find strong evidence that trait-level emotion reappraisal is associated with truth discernment performance. No other interaction or main effects were significant. When including suppression in the models, we found a main effect of headline veracity, $\mathrm{b}=$

\footnotetext{
${ }^{4}$ In Study 1, we did not find evidence that trait level emotional reappraisal correlates with better discernment, and emotion suppression was even associated with worst discernment (see supplementary materials for details).
} 
$2.58, \mathrm{p}<0.0001$, suppression, $\mathrm{b}=0.31, \mathrm{p}<0.0001$, and concordance, $\mathrm{b}=0.69, \mathrm{p}<0.0001$.

We also found a significant negative interaction between suppression and veracity, $b=-0.13$, $\mathrm{p}<0.0001$, such that suppression was associated with worse truth discernment. This suggests that habitual use of emotion regulation techniques might not help people discern the truth of news headlines. However, note that we only measured trait level emotion regulation and not state emotion regulation; depending on the task, a lack of correlation between state and trait emotion regulation has been often observed (Ehring et al., 2010). In other words, this data does not speak of the potential effect of making people regulate their emotions while they are evaluating specific headlines.

\section{Study 2}

\section{Methods}

Participants. We preregistered 1000 participants in the 3-condition between-subject design. In total, 1007 participants took part in this experiment, who were recruited through MTurk, and completed the experiment online. 338 people took part in the Reappraisal condition (144 females, 190 males and 4 others, Mean age $=37.7$ years, $S D=10.7$ years) 113 were Republican and 224 were Democrat leaning, while 1 did not respond. 331 additional people took part in the Suppression condition (130 females, 200 males and 1 other, Mean age = 38.1 years, $\mathrm{SD}=10.7$ years), among whom 209 were Democrat-leaning, 119 were

Republican-leaning and 3 did not respond. Finally, 338 people took part in the control condition (135 females, 199 males and 4 others, Mean age $=37.5$ years, $\mathrm{SD}=11.3$ years), among whom 213 were Democrat-leaning, 123 were Republican-leaning and 2 did not respond. The preregistration of the experiment is available at:

http://aspredicted.org/blind.php?x=n2vv9s

\section{Materials and Procedure}

We used the same set of headlines as in Study 1. Participants received 16 headlines altogether, in a random order - just as in Study 1. After each headline was presented, participants were asked three questions, always in the following order: perceived accuracy, willingness to click on the headline, and willingness to share the headline. The questions asked were the same as in Study 1.

Participants were randomly assigned to one of the three between-subject conditions: Control, Emotional Suppression, or Emotional Reappraisal. In the treatment conditions (emotional suppression and emotional reappraisal conditions) participants received the same instructions before the first trial of the experiment and after every fourth trial was passed. For both conditions, we took the instructions from Lee et al. (2013). These procedures have been shown to effectively evoke emotion regulation strategies, reduce emotional responses and induce deliberation in moral dilemmas (Feinberg et al., 2012), economic games (van't Wout et al., 2010) and policy support (Lee et al., 2013). 
We used the following instructions for the emotional suppression condition:

"As you view and read the headlines, if you have any feelings, please try your best not to let those feelings show. Read all of the headlines carefully, but try to behave so that someone watching you would not know that you are feeling anything at all."

We used the following instructions for the emotion reappraisal condition:

"As you view and read the headlines, please try to adopt a detached and unemotional attitude. Please try to think about what you are reading objectively. Read all of the headlines carefully, but please try to think about what you are seeing in such a way that you feel less emotion."

Note that this prompt is different from Lee et al.'s instructions as those authors explicitly motivated people to feel more positive emotion. From Study 1, we know that even positive emotions, such as happiness, makes people more susceptible to believe in fake news. Hence, we decided to change the prompt in a way to encourage people to try to feel less emotion overall.

At the end of the experiment, participants were presented with the 7 item Cognitive Reflection Task questionnaire, and various questions regarding demographics and political orientation. As per our pre-registration, we classified participants into "Democrats" and "Republicans" based on the question: "Which of the following best describes your political preference? (Strongly Democrat/Democrat/Lean Democrat/ Lean Republican/Republican/Strong Republican)". We used this classification to define which headlines were "politically concordant" and "politically discordant". To be consistent with Study 1, we also measured ideology and, as robustness test, conducted our analysis using ideology to classify political concordance and discordance. We found no meaningful difference in our results using this measure.

Statistical procedure. We used mixed-effect logistic regression models with items and subjects as random intercepts, and experimental condition $(0=$ condition $\mathrm{A}, 1=$ condition $\mathrm{B}$; which condition counts as $\mathrm{A}$ or $\mathrm{B}$ depends on the analysis $)$, veracity of headlines $(-0.5=$ false, $0.5=$ true $)$, political concordance $(-0.5=$ discordant, $0=$ neutral, $0.5=$ concordant $)$ as fixed effects. To define the random structure we started with the maximal model (allowing for the intercept to vary over subjects and headlines, and all the fixed effects to vary over headlines and subjects), and if not converged, we started decreasing its complexity. We summarized the most complex model that converged, if more, similarly complex model converged, we used the best fitting model based on AIC. 


\section{Results}

Figure 3 summarizes the results. As pre-registered, we first tested whether or not there was any significant difference between the two test conditions in the effectiveness of reducing perceived accuracy for fake news. As fixed effects, we added concordance, headline veracity and treatment condition. The model with the most complex random structure that converged allowed the intercept to vary among subjects and headlines, and allowed for the effect of headline veracity and concordance to vary over subjects and the effect of condition and concordance to vary over items. Emotion regulation techniques did not differ in their effectiveness on increasing discernment; treatment variable had no mean effect, $b=0.06, p=$ 0.65 , it had no significant interaction with headline veracity, $b=-0.24, p=0.2$, no interaction with concordance, $\mathrm{b}=-0.1, \mathrm{p}=0.38$, and no three-way interaction, $\mathrm{b}=0.04, \mathrm{p}=0.87$.

As a next step, since we found no difference between treatment conditions, we compared the pooled treatment conditions with the control condition. In this comparison, the model with the most complex random structure that converged and had the best fit (AIC $=16834$ ) allowed for the intercept to vary between items and subjects, allowed for the effect of headline veracity to vary over subjects, and allowed the effect of concordance to vary over items and subjects. We found a significant main effect of concordance $b=0.4, p=0.02$ (people were more likely to believe in concordant headlines) and headline veracity $b=2.2, p$ $<0.0001$ (real headlines were perceived as more accurate then fake headlines), but the main effect of treatment condition was not significant, $b=-0.17, p=0.08$. We did not find a significant interaction between headline veracity and condition $b=0.24, p=0.08$, there was no significant interaction between treatment and concordance, $b=-0.008, p=0.93$, and no significant three-way interaction between treatment, veracity, and concordance, $b=0.33, p=$ 0.06 .

Based on visual inspection of Figure 6, we exploratively compared each treatment condition to the control condition separately. We found no interaction between headline veracity and reappraisal treatment, $b=0.12, p=0.41$, but we found a significant interaction between veracity and suppression treatment, $b=0.34, p=0.02$. However, since this analysis was not preregistered and we made a data-driven decision to run the analysis, these results should be interpreted with caution. 


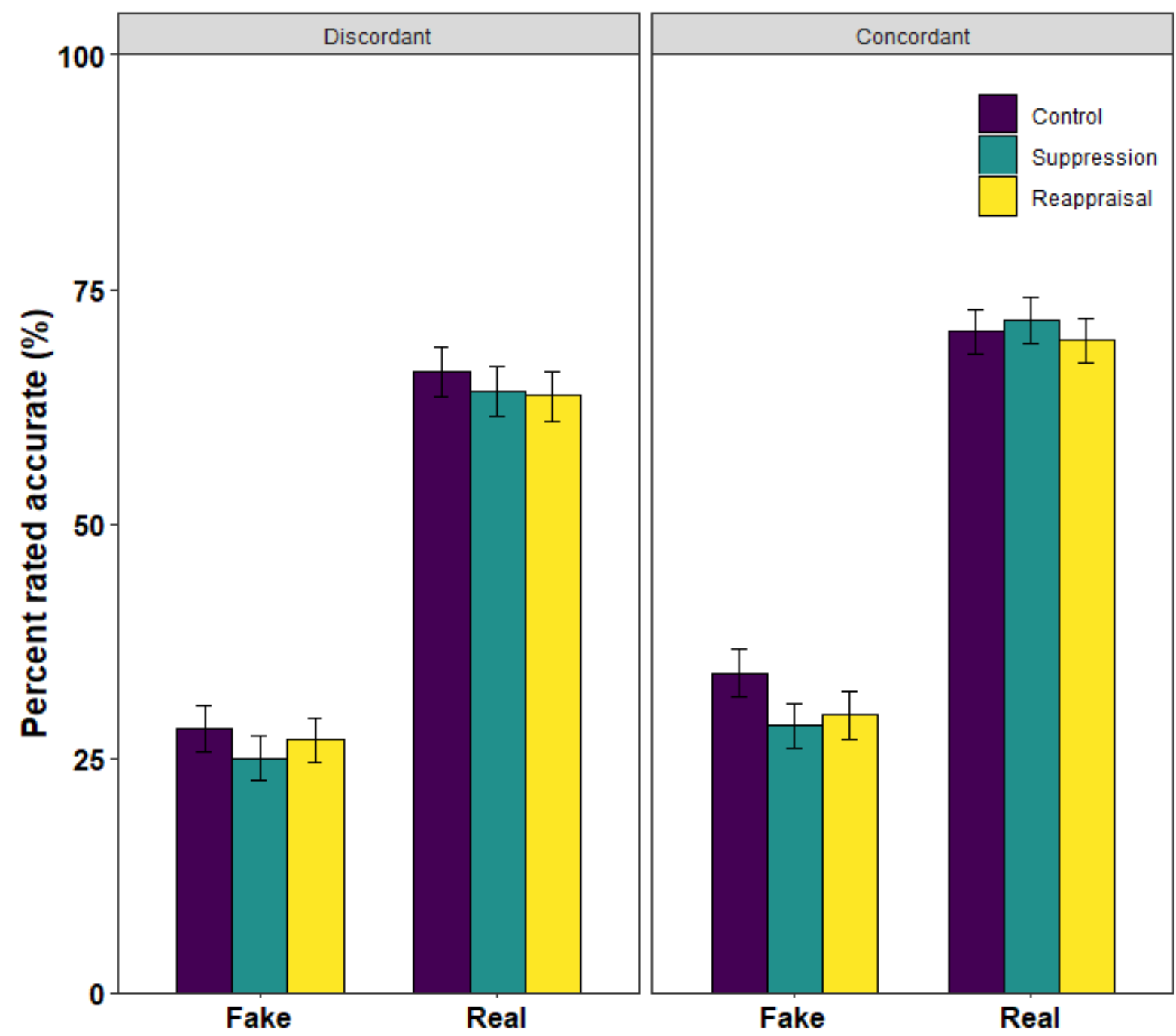

Figure 6. The figure shows perceived accuracy averages as a function of headline's concordance, veracity and experimental condition. Error bars are 95\% confidence intervals.

These results are rather inconclusive. We did not find evidence for the critical two way interaction, but there seemed to be a tendency toward a rather small effect of emotion suppression. The effect was so small that it was possible that we simply did not have sufficient power to detect it. Hence, we conducted two replication studies with larger samples.

\section{Study 3 - 4}

Since emotion suppression had a bigger effect on truth discernment in Study 2, we only used the emotion suppression prompt in Studies 3 and 4. Prior to these studies we conducted a simulation-based sample size estimation using the SimR package in R (Green \& MacLeod, 2016), based on the observed effect size for the emotion suppression effect, while still accounting for the random effect structure we a priori observed. This analysis suggested that 
we would need 3000 participants altogether (1500 per condition) to achieve power above $90 \%$. This is the sample size we recruited for both experiments.

\section{Methods}

\section{Participants}

Study 3. In total, 3001 participants took part in this experiment, who were recruited through MTurk, and completed the experiment online. 1502 people took part in the Suppression condition ( 712 females, 778 males and 12 others, Mean age $=41.3$ years, $\mathrm{SD}=13.1$ years) 601 were Republican and 894 were Democrat leaning. 1499 additional people took part in the Control condition ( 704 females, 778 males and 17 others, Mean age $=40.9$ years, SD $=$ 12.7 years), among whom 876 were Democrat-leaning, 617 were Republican-leaning. Study 3 was conducted in September, 2020. The preregistration of the experiment is available at: https://aspredicted.org/blind.php? $\mathrm{x}=6 \times 5 \mathrm{ux} 8$

Study 4. In total, 3032 participants took part in this experiment, who were recruited through MTurk, and completed the experiment online. 1520 people took part in the Suppression condition (746 females, 742 males and 21 others, Mean age $=41.2$ years, $\mathrm{SD}=13.2$ years)

561 were Republican and 946 were Democrat leaning. 1512 additional people took part in the Control condition ( 724 females, 759 males and 17 others, Mean age $=41.7$ years, $\mathrm{SD}=12.8$ years), among whom 946 were Democrat-leaning, 549 were Republican-leaning. Study 4 was conducted in January, 2021.

\section{Procedure}

Study 3 procedure. We used the exact same procedure as in Study 2, with the exception that the Reappraisal condition was not administered.

Study 4 procedure. We used newer headlines taken from Pennycook, Binnendyk, et al., (2020). They measured 3 types of emotional reactions to each headline: "anger", "funny" and "anxiety". Out of the many headlines they tested, we selected both Republican and Democrat consistent fake and real headlines that scored highest in these emotion categories (i.e., two highest scores in the "funny" category within Democrat consistency - fake etc). Altogether we used 24 headlines, out of which participants were randomly assigned to 16; 4 in each cell (partisan consistency; and headline veracity).

We further simplified the experiment and only measured perceived accuracy, and not sharing or clicking intentions. Furthermore, in order to further assure that participants follow and read the instructions in the emotion regulation condition, participants received the prompt on top of each individual headline. 
Finally, we also added two attention check questions because of concerns about inattention on MTurk (Arechar \& Rand, 2021): "Puppy is to dog as kitten is to?" and "People are very busy these days and many do not have time to follow what goes on in the government. We are testing whether people read questions. To show that you've read this much, answer both "extremely interested" and "very interested": [5 options]"

\section{Results}

Figure 7 summarizes the results for both studies. In Study 3, the model allowed for the intercept to vary between items and subjects, allowing for the effect of veracity and concordance to vary of subjects and and concordance to vary over items. In Study 3, we found no significant interaction between headline's veracity and emotion regulation condition, $\mathrm{b}=-0.05, \mathrm{p}=0.46$, nor a three-way interaction between emotion regulation, concordance and veracity, $\mathrm{b}=0.14, \mathrm{p}=0.14$, and emotion regulation also had no main effect, $\mathrm{b}=0.00001, \mathrm{p}=0.99$. In Study 4 , the model allowed for the intercept to vary between items and subjects, allowing for the effect of concordance and veracity to vary over subjects and items. There was no main effect of the emotion regulation condition, $b=0.03, p=0.55$, no interaction between veracity and emotion regulation condition, $b=-0.12, p=0.16$, and no three-way interaction between condition, veracity and concordance, $b=-0.05 ; p=0.64$. This suggests that emotion regulation had no effect on truth discernment.
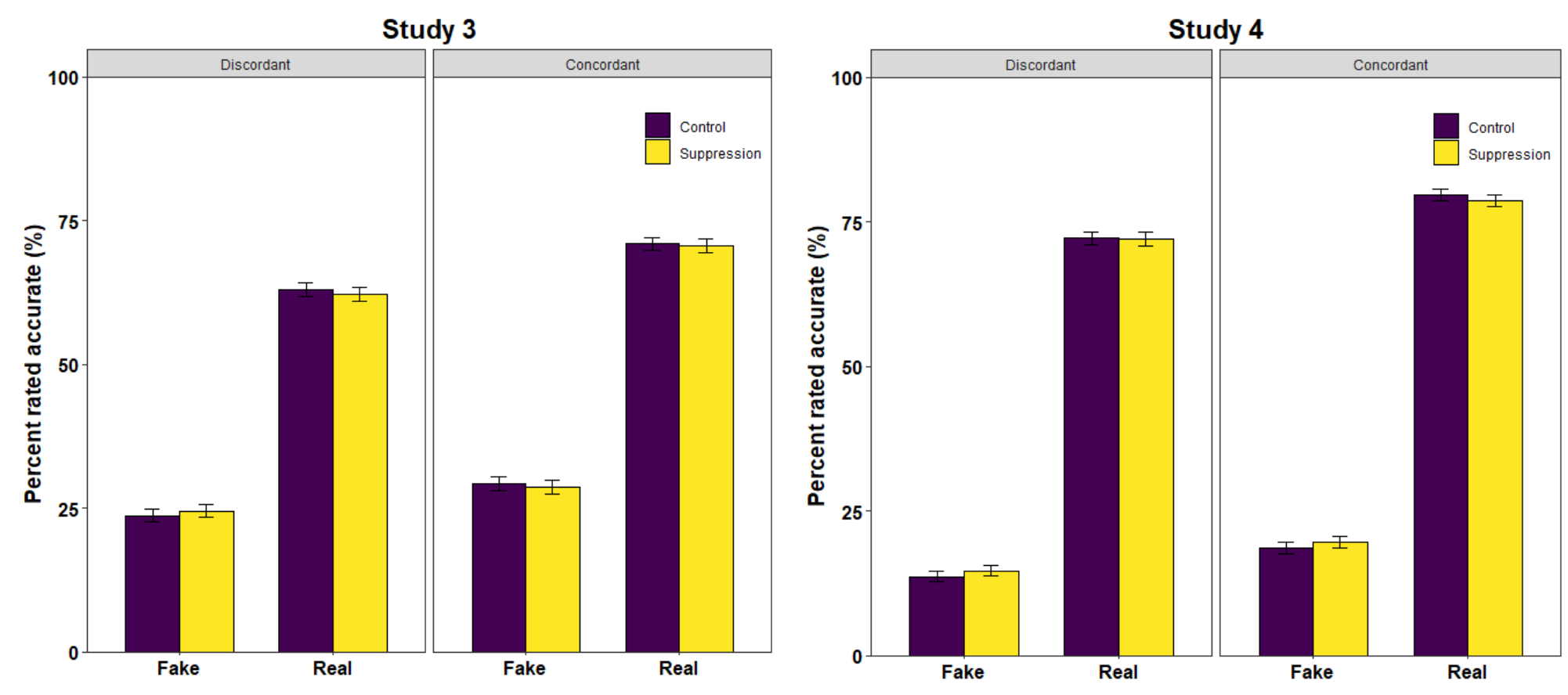

Figure 7. The figure shows perceived accuracy averages as a function of headline's concordance, veracity and experimental condition both on Study 3 (left) and Study 4 (right). Error bars are $95 \%$ confidence intervals. 
One objection to these results could be that Mturkers simply did not pay any attention to the manipulation prompt. In both Study 3 and Study 4, we used Mturkers who produced high quality data in the past; participants were only allowed to enter the experiment if they had a HIT approval rate of $98 \%$ and who at least completed 100 HITs. Furthermore, in Study 4, we also added two attention check questions to the beginning of the experiment (before the actual headlines were presented). When excluding any participants who failed any of the two attention check questions, 186 people altogether (i.e., 94\% of participants passed the attention check), we found similar results: Most importantly, neither the two-way interaction between headline veracity and treatment condition, $b=-0.11, p=0.21$, nor the three-way interaction, between veracity, concordance and treatment condition, $b=-0.07, p=0.5$, were significant.

We also conducted a pooled analysis in which we pooled together all studies in which we had the emotion regulation prompt (that is, we excluded the emotion reappraisal condition from Study 2). Again, even with a sample size of 6702 participants, we found no evidence that emotion suppression helped people distinguish fake from true news headlines; the veracity and condition interaction was not significant, $b=-0.03, p=0.59$, and neither was the three-way interaction between concordance, emotion suppression condition and headline veracity, $b=0.14, p=0.054$. The random effect structure of this model that converged included the random intercepts of subjects and items and allowed for variation of veracity and concordance over subjects and concordance over items.

One reason we might not have found any effect of emotion regulation is that the techniques we used to make people regulate their emotional response are disproportionately better at decreasing anger than any other emotion. Since we observed a positive association between anger and truth discernment, a potential decrease in anger might have led to worse truth discernment, while a decrease in any other emotion might have led to better discernment - and overall, the two effects could have cancelled each other out. To investigate this possibility, we classified each news headline from Study 1, as "high anger" and "low anger" items, based on a median split on how many participants reported feeling anger for each headline (separately by concordance). When analysing high anger items from Study 2 and Study 3, surprisingly, we found a significant three-way interaction between condition, veracity and concordance, $b=0.27, p=0.02$, such that emotion regulation increased discernment for concordant items, $b=0.19, p=0.04$, but not for discordant items, $b=-0.1, p$ $=0.23$, (and the two way interaction between condition and veracity was not significant, $\mathrm{b}=$ $0.06, p=0.39$, and no main effect of condition, $b=0.004, p=0.93)$. On low anger items we found no significant effect of emotion regulation condition (no condition $\mathrm{x}$ veracity $\mathrm{x}$ concordance interaction, $\mathrm{b}=-0.03, \mathrm{p}=0.83$, no condition $\mathrm{x}$ veracity interaction, $\mathrm{b}=0.004, \mathrm{p}$ $=0.96$, no main effect of condition, $b=-0.07, p=0.1$ ). This suggests that our null effects were not caused by emotion regulation techniques preferentially decreasing anger.

\section{Discussion}


This study tested the idea that headlines which are more emotionally evocative are also harder to discern as true or false. In the first study, we found that emotional response, in general, at the headline level were associated with decreased truth discernment. These correlational results served as the impetus for the second set of studies to test the effectiveness of an intervention designed to improve discernment. In this second set of studies, however, we did not find compelling evidence that an emotion regulation intervention helps people correctly discern the truth (although we did find some limited evidence that emotion regulation might help against politically concordant false headlines that are anger-inducing; nevertheless, this was an unexpected finding of a non-preregistered analysis, so should be interpreted with caution).

There have been at least two major theories that try to capture how emotions affect thought. One line of research argues that certain emotions, such as fear and sadness make people more deliberative, while others, such as anger or happiness, make people less deliberative (Forgas, 2013; Holland et al., 2012; Lerner \& Tiedens, 2006). It has been shown that deliberation positively affects discernment (Bago et al., 2020). Hence, if certain emotions induce deliberation people will be more discerning (or if they induce intuition, they will be less discerning). Our findings provide evidence to the contrary, where we observed no association or worse discernment for the majority of emotions except anger. Anger, which is said to be one emotion that induces intuition (Lerner \& Tiedens, 2006), is actually correlated with improved discernment, in all of our dependent variables; perceived accuracy, sharing and clicking intentions. However, when interpreting specific results, we should keep in mind that our first study is purely correlational.

The second set of influential theories suggest that people use an "affective heuristic" (Slovic et al., 2007). That is, people use their affective evaluation of a stimulus to make a judgment about the stimulus. If the affective evaluation of a headline is negative (e.g., they feel negative emotions) they will be less likely to believe it (regardless of veracity). This theory could help us explain the main "effect" of happiness and surprise on perceived accuracy: happiness and surprise were associated with increased overall belief regardless of veracity, which is in agreement with this theory. However, sadness had a positive "effect" on overall belief levels; even though it is a negative emotion. It is also unclear how this theory could explain the emotional effects on truth discernment. It is possible that emotions have at least two types of effects on truth judgement; their positive-negative valence increases or decreases overall belief (under some unknown conditions), while they could also make deliberative engagement more or less likely. Future research and theorizing on emotion and thought in truth discernment should address this question.

Since misinformation is a global phenomenon, it is critical to see how generalizable our results are across countries. This is particularly true given well-documented cross-cultural variations in emotional responses and emotion regulation more broadly (e.g., Mesquita \& Frijda, 1992). Consistent with our findings, there is some evidence that emotions, in general, are associated negatively with truth discernment in Nigeria (Rosenzweig et al., 2021). On the 
other hand, contrary to our results, there has also been some evidence that anger is negatively associated with truth discernment in both South Korea (Han et al., 2020) and negatively associated with overall belief in Nigeria (Rosenzweig et al., 2021) in the Covid-19 context, whereas we observed a positive association in the U.S between anger and truth discernment. Although it is yet unclear if this difference was created by the difference in contexts (Covid-19 vs politics) or reflects cultural variation, future research should address this question.

Although emotions seem to be observationally associated with truth discernment abilities, we found little evidence that an intervention that directly tried to decrease emotional processing was helpful. One caveat of our research is that we had no independent manipulation check (i.e., does our manipulation indeed induce emotion regulation?) but given the many successes these methods have been used to decrease emotional effects on decision making, it seems unlikely that the manipulation did not work. Second, in Study 1, we also had correlational evidence that shows no significant positive associations between spontaneous emotion regulation (i.e., the frequency people use certain emotion regulation techniques in their daily lives) and truth discernment performance. It seems more likely that the canonical emotion regulation techniques that we tested seem to be ineffective at helping people identify fake news. One potential reason is that inducing emotion regulation made people focus on their emotions, and what emotions the headline is making them feel, and got distracted from other cues that would potentially help them discern what was true or false. Future research could test techniques that induce emotion regulation, but besides making participants cooler, help them re-focus their attention on the veracity of headlines. While these could be an interesting theoretical question for future research, using emotion regulation in its current form is unlikely to help us in the battle against misinformation.

\section{Acknowledgement}

We gratefully acknowledge funding from ANR grant ANR-17-EURE-0010 (Investissements d'Avenir program, BB,LR), ANR Labex IAST (BB, LR).

References:

Arechar, A. A., \& Rand, D. G. (2021). Turking in the time of COVID. Behavior Research Methods, 1-5.

Bago, B., Rand, D. G., \& Pennycook, G. (2020). Fake news, fast and slow: Deliberation reduces belief in false (but not true) news headlines. Journal of Experimental 
Psychology: General.

Barr, R. A. (2019). Fake news grabs our attention, produces false memories and appeals to our emotions. The Conversation.

https://theconversation.com/fake-news-grabs-our-attention-produces-false-memories -and-appeals-to-our-emotions-124842

Berinsky, A. J. (2017). Rumors and health care reform: Experiments in political misinformation. British Journal of Political Science, 47(2), 241-262.

Brady, W. J., Wills, J. A., Jost, J. T., Tucker, J. A., \& Van Bavel, J. J. (2017). Emotion shapes the diffusion of moralized content in social networks. Proceedings of the National Academy of Sciences, 114(28), 7313-7318.

Ehring, T., Tuschen-Caffier, B., Schnülle, J., Fischer, S., \& Gross, J. J. (2010). Emotion regulation and vulnerability to depression: Spontaneous versus instructed use of emotion suppression and reappraisal. Emotion, 10(4), 563.

Ekman, P. (1992). An argument for basic emotions. Cognition \& Emotion, 6(3-4), 169-200.

Elfenbein, H. A., \& Ambady, N. (2002). On the universality and cultural specificity of emotion recognition: A meta-analysis. Psychological Bulletin, 128(2), 203.

Evans, J. S. B. (2012). Spot the difference: Distinguishing between two kinds of processing. Mind \& Society, 11(1), 121-131.

Evans, J. S. B., \& Stanovich, K. E. (2013). Dual-process theories of higher cognition advancing the debate. Perspectives on Psychological Science, 8(3), 223-241.

Feinberg, M., Willer, R., Antonenko, O., \& John, O. P. (2012). Liberating reason from the passions: Overriding intuitionist moral judgments through emotion reappraisal. Psychological Science, 23(7), 788-795.

Forgas, J. P. (2013). Don't worry, be sad! On the cognitive, motivational, and interpersonal benefits of negative mood. Current Directions in Psychological Science, 22(3), $225-232$. 
Frank, M. G., \& Stennett, J. (2001). The forced-choice paradigm and the perception of facial expressions of emotion. Journal of Personality and Social Psychology, 80(1), 75.

Frederick, S. (2005). Cognitive reflection and decision making. The Journal of Economic Perspectives, 19(4), 25-42.

Green, P., \& MacLeod, C. J. (2016). SIMR: an R package for power analysis of generalized linear mixed models by simulation. Methods in Ecology and Evolution, 7(4), 493-498.

Grinberg, N., Joseph, K., Friedland, L., Swire-Thompson, B., \& Lazer, D. (2019). Fake news on twitter during the 2016 US Presidential election. Science, 363(6425), 374-378.

Gross, J. J. (1998). Antecedent-and response-focused emotion regulation: Divergent consequences for experience, expression, and physiology. Journal of Personality and Social Psychology, 74(1), 224.

Gross, J. J. (2002). Emotion regulation: Affective, cognitive, and social consequences. Psychophysiology, 39(3), 281-291.

Gross, J. J., \& John, O. P. (2003). Individual differences in two emotion regulation processes: Implications for affect, relationships, and well-being. Journal of Personality and Social Psychology, 85(2), 348.

Gutierrez, R., \& Giner-Sorolla, R. (2007). Anger, disgust, and presumption of harm as reactions to taboo-breaking behaviors. Emotion, 7(4), 853 .

Han, J., Cha, M., \& Lee, W. (2020). Anger contributes to the spread of COVID-19 misinformation. Harvard Kennedy School Misinformation Review, 1(3).

Holland, R. W., Vries, M. de, Hermsen, B., \& Knippenberg, A. van. (2012). Mood and the attitude-behavior link: The happy act on impulse, the sad think twice. Social Psychological and Personality Science, 3(3), 356-364.

Langner, O., Dotsch, R., Bijlstra, G., Wigboldus, D. H., Hawk, S. T., \& Van Knippenberg, A. D. (2010). Presentation and validation of the Radboud Faces Database. Cognition and Emotion, 24(8), 1377-1388. 
Lazer, D. M., Baum, M. A., Benkler, Y., Berinsky, A. J., Greenhill, K. M., Menczer, F., Metzger, M. J., Nyhan, B., Pennycook, G., \& Rothschild, D. (2018). The science of fake news. Science, 359(6380), 1094-1096.

Lee, J. J., Sohn, Y., \& Fowler, J. H. (2013). Emotion regulation as the foundation of political attitudes: Does reappraisal decrease support for conservative policies? PloS One, 8(12), e83143.

Lerner, J. S., \& Tiedens, L. Z. (2006). Portrait of the angry decision maker: How appraisal tendencies shape anger's influence on cognition. Journal of Behavioral Decision Making, 19(2), 115-137.

Lynch, M. P. (2019). Do We Really Understand "Fake News"? The New York Times. https://www.nytimes.com/2019/09/23/opinion/fake-news.html

Martel, C., Pennycook, G., \& Rand, D. (2020). Reliance on emotion promotes belief in fake news. Cognitive Research: Principles and Implications, 5(1), 1-20.

McLaughlin, T. (2018). How whatsapp fuels fake news and violence in India. Wired (Dec 2018).

https://www.wired.com/story/how-whatsapp-fuels-fake-news-and-violence-in-india/

Mesquita, B., \& Frijda, N. H. (1992). Cultural variations in emotions: A review. Psychological Bulletin, 112(2), 179.

Pennycook, G., Binnendyk, J., Newton, C., \& Rand, D. (2020). A practical guide to doing behavioural research on fake news and misinformation.

Pennycook, G., Epstein, Z., Mosleh, M., Arechar, A. A., Eckles, D., \& Rand, D. G. (2021).

Shifting attention to accuracy can reduce misinformation online. Nature.

Pennycook, G., \& Rand, D. G. (2019). Lazy, not biased: Susceptibility to partisan fake news is better explained by lack of reasoning than by motivated reasoning. Cognition, 188, $39-50$.

Pennycook, G., \& Rand, D. G. (2021). The psychology of fake news. Trends in Cognitive 
Sciences.

Rosenzweig, L. R., Bago, B., Berinsky, A. J., \& Rand, D. G. (2021). Happiness and surprise are associated with worse truth discernment of COVID-19 headlines among social media users in Nigeria. Harvard Kennedy School Misinformation Review.

Slovic, P., Finucane, M. L., Peters, E., \& MacGregor, D. G. (2007). The affect heuristic. European Journal of Operational Research, 177(3), 1333-1352.

Thomson, K. S., \& Oppenheimer, D. M. (2016). Investigating an alternate form of the cognitive reflection test. Judgment and Decision Making, 11(1), 99-113.

van't Wout, M., Chang, L. J., \& Sanfey, A. G. (2010). The influence of emotion regulation on social interactive decision-making. Emotion, 10(6), 815.

Vosoughi, S., Roy, D., \& Aral, S. (2018). The spread of true and false news online. Science, 359(6380), 1146-1151. 